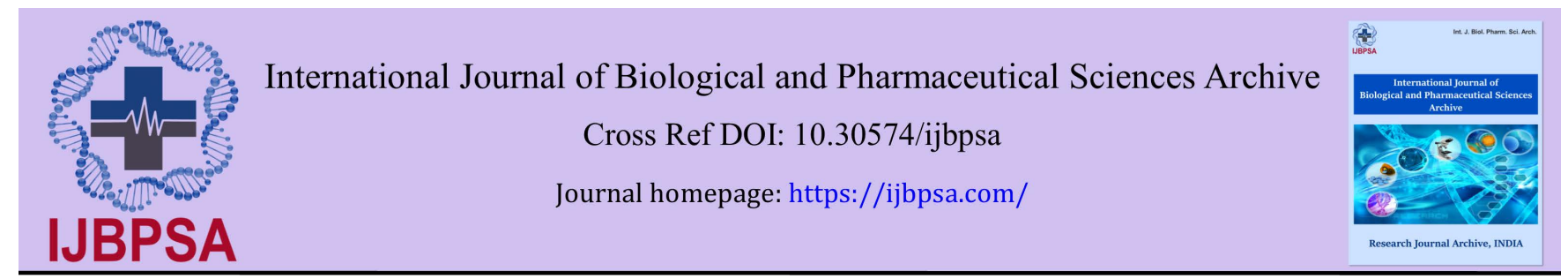

(RESEARCH ARTICLE)

\title{
Atomic kernels as waves and catastrophes
}

\author{
Gudrun Kalmbach HE*
}

MINT, PF 1533, D-86818 Bad Woerishofen, Germany.

International Journal of Biological and Pharmaceutical Sciences Archive, 2021, 01(02), 062-067

Publication history: Received on 24 January 2021; revised on 28 February 2021; accepted on 02 March 2021

Article DOI: https://doi.org/10.30574/ijbpsa.2021.1.2.0020

\begin{abstract}
The presentation of atomic kerrnels as particles requires for the physics duality principle that they get a wave description. This is due to presenting the SU (3) GellMann matrix space by octonians which are obtained by doubling the spacetime quaternions. Their multiplication table is different from the SU (3) matrices. The third presentation of this space is a complex 4-dimensional space where the real spacetime coordinates of a 4-dimensional Euclidean Hilbert space R4 are extended to C4. For getting from Deuteron Cooper pairs NP of a neutron and proton atomic kernels AK, the wave package superpositions for AK need the mass defect of neutrons where kg is changed to inner speeds or interaction energies. For kg octonians have a GF measuring base triple as Gleason operator. Using a projective geometrical norming, $\mathrm{C} 4$ is normed to $\mathrm{CP}^{3}$, a projective 3-dimensional space. Its cell $\mathrm{C}^{3}$ has spacetime coordinates $\mathrm{C}^{2}$, extended by an Einstein energy plane $\mathrm{z} 3=(\mathrm{m}, \mathrm{f}), \mathrm{m}$ mass, $\mathrm{f}=1 / \Delta \mathrm{t}$ frequency where mass can be transformed into $\mathrm{f}$ by using $\mathrm{mc}^{2}=\mathrm{hf}$. If $\mathrm{C}^{3}$ is presented as a real space R6, it can be real projective normed to a real projective space P5 for the field presentation of AK. As field the NP's have then a common group speed for AK wave packages superpositions with which $\mathrm{AK}$ moves in spacetime $\mathrm{C}^{2}$ and also a presentation as a $\Psi$ wave. As probability distribution where they can be energetically found in space serves $\Psi^{*} \Psi$.
\end{abstract}

Keywords: Atomic Kernels; Fiber Bundles; Catstrophes

\section{Fiber Bundles}

Both, the weak and strong intercation have fiber bundles for particle descriptions. Waves have no influence on this. The weak interaction has, also for electromagnetism, the Hopf geometry with the SU (2) symmetry. The twisted fiber bundle maps $\mathrm{R} 4$ down to $\mathrm{R}^{3}$ and the unit sphere $\mathrm{S}^{3}$ in $\mathrm{R} 4$ down to the complex Riemannian sphere $\mathrm{S}^{2}$ in $\mathrm{R}^{3}$. Normed is the time coordinate. In a stereogrpahic projection $\mathrm{S}^{2}$ is mapped to a complex plane $\mathrm{C}$ with coordinates $\mathrm{w}=\mathrm{x}+\mathrm{iy}$. Normed is the real z-coordinate. If $\mathrm{R} 4$ is presented in quaternion matrix notation as complex $\mathrm{C}^{2}$ space with coordinates $\mathrm{z} 1=\mathrm{z}+\mathrm{ict}, \mathrm{z} 2$ $=\mathrm{x}+\mathrm{iy}$, the $\mathrm{C}$ coordinate is $\mathrm{w}=\mathrm{z} 2 / \mathrm{z} 1$ for $\mathrm{z} 1 \neq 0$ and $\mathrm{z} 1=0$ is the projective point $\infty$ in $\mathrm{S}^{2}$ as northpole for the stereographic projection. The Hopf map gives the possibility to add to an AK several electrons in an atoms shell. Their probability distribution $\Psi^{*} \Psi$ uses a mass defect of electrons for getting the atoms group speed for the waves $\Psi$ momentum. The main quantum numbers for electrons location on atoms Bohr shells are obtained by setting attracting Coulomb force equal to the centrifugal (escape) gravity force acting on the electron. Since angular momentum is spin quantized in this case, the quantization of spin by natural numbers sets the main quantum numbers. It determines the different Bohr radii for the electron. When they jump in between radii locations, electromagnetic waves are released or absorbed as spectral series from the atom. This possibility arises later in the development of a dark universe, not after a big bang. The former mentioned norming of octonians is reversed. The complex space (z1, z2, z3) is extended by the complex cross product to octonians as C4 with adding $\mathrm{z} 4=\mathrm{z} 1 \mathrm{xz} 2 \mathrm{xz} 3$.

\footnotetext{
${ }^{*}$ Corresponding author: Gudrun Kalmbach H.E

MINT, PF 1533, D-86818 Bad Woerishofen, Germany.
} 
For the use of the Hopf map in the projective normed $\mathrm{C}^{3}$ cell of $\mathrm{CP}^{3}$, electrons can have spherical, club shaped or torus manifold geometries for their probability distribution (figure 1). The Hopf projection allows spheres $S^{2}$ as a shape, the inverse Hopf map the torus blow up in $\mathrm{S}^{3}$ from a latitude circle S1 with a quantized main number Bohr radius. S1 is blown up by a fiber $\mathrm{S} 1$ to the torus location $\mathrm{S} 1 \mathrm{xS} 1$ in $\mathrm{S}^{3}$. In $\mathrm{S}^{2}$ the electron rotates with the latitude circle clockwise cw, in $\mathrm{S}^{3}$ with the torus $\mathrm{cw}$ about a central axis of the torus. The spherical clubs arise through the other quantum numbers. The nth roots of unity determine the leaning angle for the electrons towards the z-axis by repulsion (figure 1 at right). The Heisenberg uncertainty angle/angular momentum requires that the spin is rotating on a cone, also the orbital angular momentum and both are vectorial added for its rotation axis. This is a dynamical geometry for the electron in an atoms wave package. It uses the SU (2) weak interaction WI quaternionic symmetry. The generating Pauli matrices define the Hopf map. They are obtained by replacing coordinates by Euler angle transformations (figure 3) which by rotation of a presenting wheel generate the axis for the Euler angle and the mathematical quaternions are computed through the Euler angles. The Hopf fiber bundle makes the electrons orbital shapes.
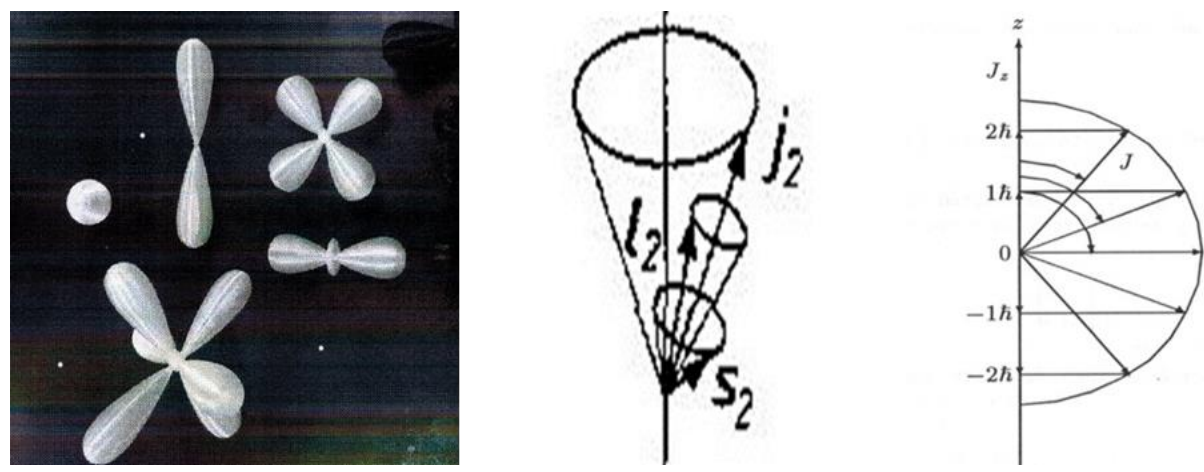

Figure 1 orbitals for electrons in an atoms shell; middle spin, orbit, rotation axes of an electron; at right electrons nth roots distribution on a shell
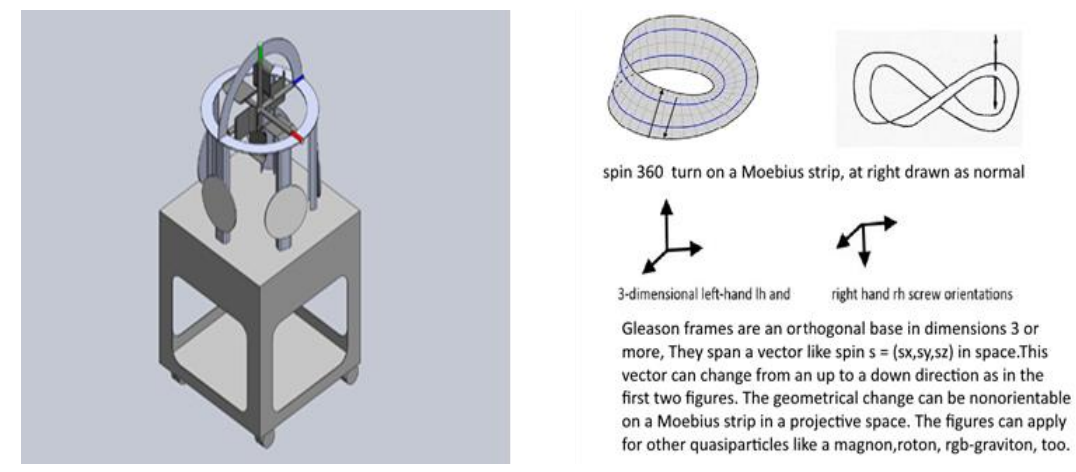

Figure 2 wheel for Euler angle rotations, generating in their xy-, xz-, yz-plane rotations the three orthogonal axes to the plane; at right the spin orthogonal base triple up/down positions, turnings are on a Moebius strip

After this dynamical geometry, for the NP Cooper pairs in an AK is responsible a SU (3) fiber bundle of the strong interaction SI. Its geometry has a topological manifold twisted bundle $\mathrm{S}^{3} \mathrm{xS} 5$, the 5 -dimensional unit sphere is in $\mathrm{C}^{3}$. The NP fiber bundle is described in several articles in the reference list [1-4]. The bundle map is g: S5 $\rightarrow \mathrm{CP}^{2}$, the complex 2dimensional inner spacetime for NP with an $S^{2}$ bounding sphere added. The $S^{3}$ factor of the manifold is projected by rgb-gravitons down to the Hopf $S^{3}$ for space presentations, in NP as a nucleon tetrahedron with an inner discrete, not wave dynamics. An SI rotor oscillation occurs. The joined proton, neutron tetrahedrons can be Cooper pair splitted in space with excitons keeping them in a isospin I3 weak WI energy exchange. The distance is bounded in an AK. If it gets too large such that the partner cannot be reached, stochastic AK decays with halflifetimes occur. For this the mass defect of neutrinos is responsible, similar to the former described one for electrons. A higher inner rotational speed or interaction energy keeps the AK together for a common group speed with which the AK wave pacckage moves in its environment. The rotational direction of spins/magnetic momentums are paired as known.

from physics. They are arranged parallel or antiparallel such that most of them are cancelled. Maybe energy, photons for instance, are emitted from a new in AK inserted Cooper pair. 


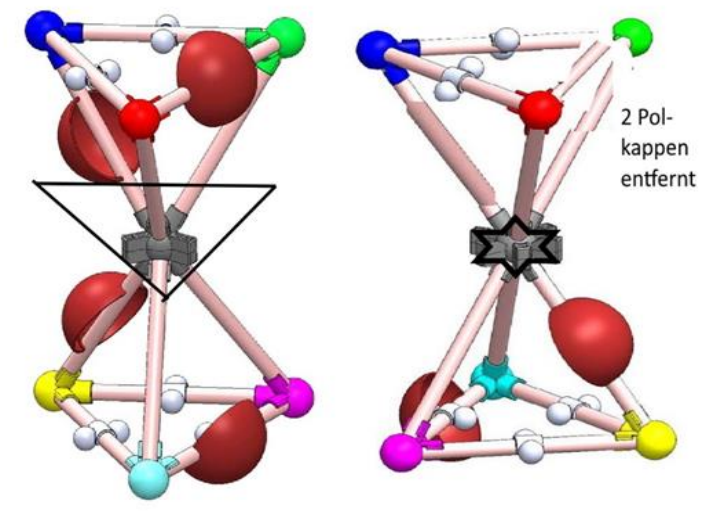

Figure 3 Deuteron fushion from two protons at left by rotation to a neutron, proton NP Cooper pair at right; the caps persent here a positron as charge of a proton; a weak isopsin exchange as an WI rotor is an inner dynamics for this where proton/neutron exchange their local position [5]

The twisted SI fiber bundle $\mathrm{S}^{3} \mathrm{xS} 5$ can be similar interpreted as the torus $\mathrm{S} 1 \mathrm{xS} 1$ for an electron. A replacement for the Hopf may can be a map gSI from in the unit sphere S9 in C5 to S5 in C3 with fiber $\mathrm{S}^{3}$. For the $\mathrm{S}^{3} \mathrm{xS} 5$ geometry in SU (3), the linear octonian or complex C4 geometry is not valid. The generating GellMann matrices $\lambda$ j are projection maps, replacing Euler angles in the SU (2) case. The vectorial written Pauli 2x2-matrices (z1 z2) are extended to 3x3-matrices (z1 z2 z3) (first matrix row) by inserting a row and column with coordinates 0 , (z1 z2 0), (z1 0 z3), ( 0 z2 z3). The effect for 8 gluons is that the extended Pauli $\sigma 3$ matrices are linearly dependent and generate a plane for two $\lambda 3, \lambda 8$ matrices. There are six gluons with two color charges and two with 6 color charges. The rgb-graviton presents the neutral color charge of all nucleons and is a superposition of three color charge whirls. The 2-gluons make the SI rotors dynamic when exchanged between paired quarks and the rgb-graviton makes the 3-dimensional nucleon tetrahedron with base a quark triangle. It is a spacial spin-like GF orthogonal base triple. The two 6-gluons make a Bohr shell atmosphere of $S^{2}$ as boundary of the nucleon/deuteron $\mathrm{CP}^{2}$ space.

\section{Catastrophes}

For the sudden changes, catastrophes apply [9-17]. They allow bifurcations and degenerate topological critical structures in manifolds (figure 4). Equations for seven basic catastrophes are shown. Beside coordinates, parameters are added which can be used as constant scalings or for projections. A projective parameter for a central (stereographic) or orthogonal (spiralic) projection has a similar parametrization. The cusp is shown at left in figure 4 in $R^{3}$ and mapped down to a (a,b) parameter plane showing two bifurcation curves with an initial common point $\mathrm{P}$. In linear form this is a pair of rays with origin $\mathrm{P}$ which define an angle. In the figure for electron distributions (figure 1 right part), this is a spherical $\theta$-angle, measured towards the z-coordinate in space. Energy systems are located on different potential planes, for instance of the 3-dimensional cusp as its upper and lower levels in space. The crictical cusp point P is in space not critical, but in its projection. Morse functions and maps are added to catastrophes (at right with equations in figure 4). The critical points Morse theory is extended by using Taylor polynomials for the potentials catastrophe equations. 


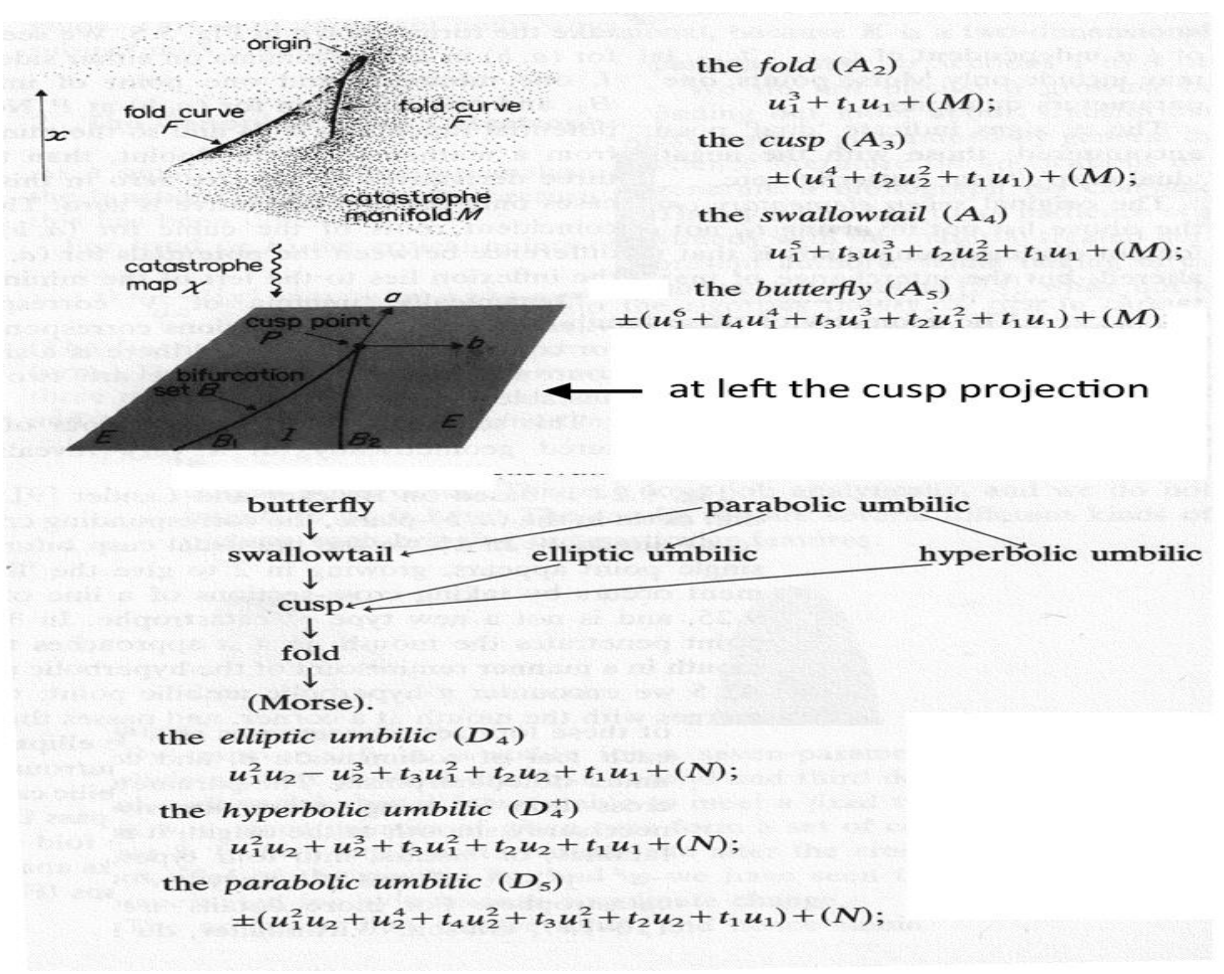

Figure 4 At left the cusp catastrophe, at right and below equations for seven catastrophes [9]

An energy bifurcation is shown in the colportage of figure 5. It can use the fold where at the tip of the fold the bifurcation is into an upper halfplane and a lower half plane for different potentials. An energy states jump is mostly in levels like this and not observed continously, it is fold sitting on a half plane (figure 5 right part).

The six generated energies have different qualities and GF orthogonal base octonian triples; 123 for 1, 257 for 5 , 246 for 2, 145 for 4, 347 for 3, 356 for 6. The GF's occur in a list called Fano memo for octonians. 126 is a GF for rgb-gravitons, an SI color charge GF. Energies can be attributed to the octonian e 0 coordinate as six color charge whirls and to the stereographic rolled U(1) coordinate 7 as electromagnetic EMI energy, light. 07 is used as a compass with e0 as an eigenvector needle and U(1) a boundary of a compass disk [7]. The needle turns with the nth roots of unitiy. If a G 2x2matrix of order 6 is used (6th roots of unity), in turning, it sets a color charge between two adjacent roots, the segmentes are cut out and folded to whirls which itself is generated as surface by a rotating eigenvector with inital point at the cones tip and $\mathrm{U}(1)$ as the cones boundary. The G matrix has as first row (1 -1) and as second row (1 0). This is a base of a projective space $[\mathrm{u}, \mathrm{v}, \mathrm{w}]$ with $\mathrm{u}=\mathrm{r}$ radius, $\mathrm{v}=\mathrm{r}-\mathrm{Rs}$, setting $\mathrm{w}=0$ allows for a Moebius transformation projective scaling $[1,(\mathrm{r}-\mathrm{Rs}) / \mathrm{r}, 0]$
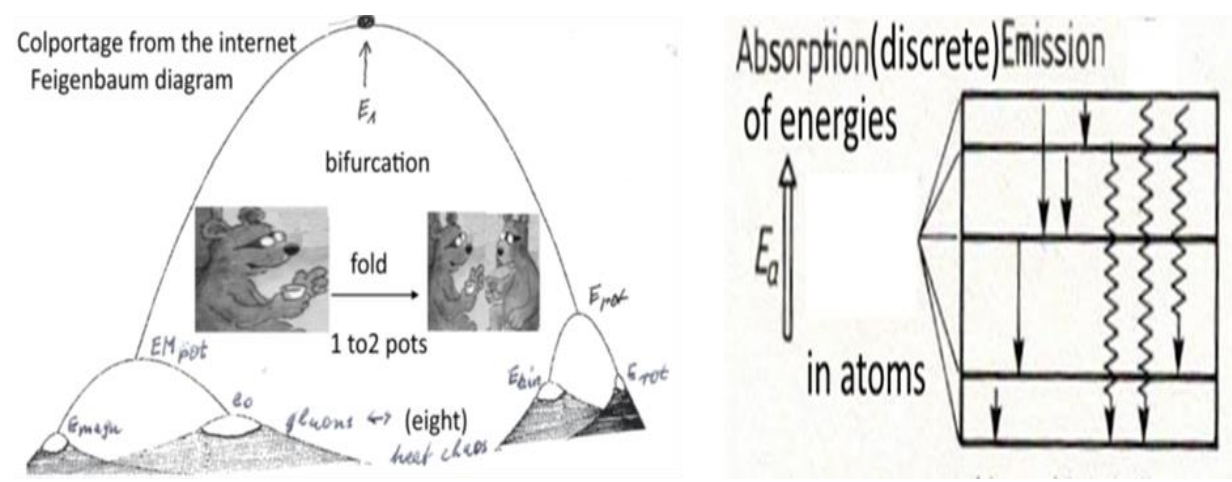

Figure 5 energy levels at right; bifurcation at left, a common octonian vector is e0 , bifurcating into an electrical and mass potential EM(pot), E(pot), in octonian coordinates indizes 1, 5, 1 is bifurcating into 2, 4 for $E$ (heat) and $E$ (magn) energies, 4 also is for time, 5 is bifuracting into kinetic and rotational energies 6, 3, the four are bifurcating into the SI eight gluons, then heat chaos is bifurcating (Feigenbaum diagram) 
for getting the Schwarzschild radius rescaling factor of Minkowski metrics time in differentials $d t^{\prime}=\mathrm{dt}^{\mathrm{cos}} \beta$ with $\sin ^{2} \beta$ $=\mathrm{Rs} / \mathrm{r}$. In rescaling time, the area of the metrics radius, time tangential plane is preserved such that gravitational GR

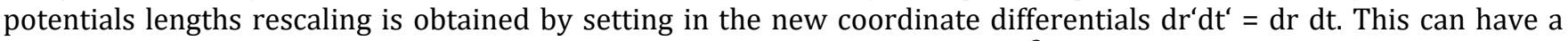
catastrophe GR generated bifurcation. For the extension of the (z1 z2 z3) $\mathrm{C}^{3}$ space to C4 it means that the octonian plane $07[\mathrm{u}, \mathrm{v}, \mathrm{w}]$ as new cross product complex coordinate $\mathrm{z} 4$ is generated. [u,v,w] is for an unsymmetrical distance measure between a small rotating system $P$ about a huge system Q (as sun for instance), having Rs as Schwarzschild radius; $|\mathrm{QP}|=\mathrm{r},|\mathrm{PQ}|=$ (r-Rs). The catastrophe parameters are for a central projection. Another interpretation for $\mathrm{G}$ is that it is the cylic time dynamics for the quark triangles symmetry D3 for the SI rotor. It arises when the nucleons tetrahedron symmetry S4 of order 24 is factorized by the CPT Klein group Z2xZ2.. C is the conjugation operator of physics, P parity, T time reversal.

Minkowski metric is also catastrophe generated. A list of quadrics bleongs to normal forms in a projective $\mathrm{P}^{2}$ space (figure 6)

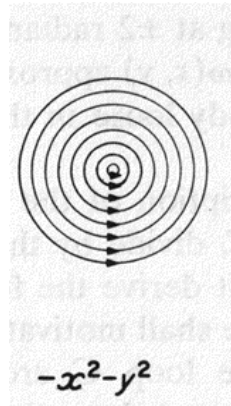

(a)

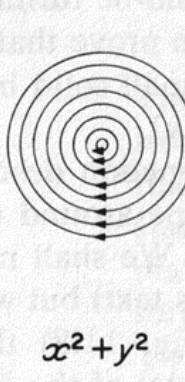

(b)

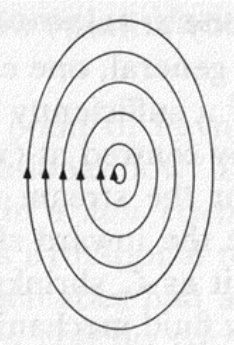

$x^{2}+\frac{1}{2} y^{2}$

(c)

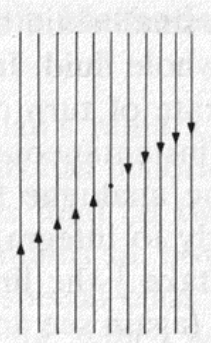

$x^{2}$

(d)
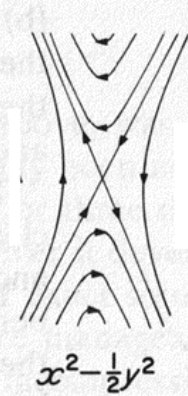

(e)

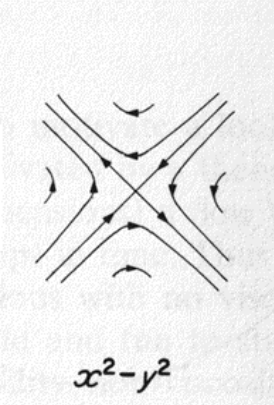

(f)

Figure 6 flows for catastrophe potentials, (f) is for the Minkowski metric with a light cone where $\mathrm{y}=\mathrm{ct}$ (time, $\mathrm{c}$ speed of light) is substituted and $\mathrm{x}^{2}=\mathrm{r}^{2}=\mathrm{x}^{2}+\mathrm{y}^{2}+\mathrm{z}^{2}$ for space coordinates

For projective shapes is added that many uses are for the real $\mathrm{P}^{3}$ normal forms. For a projective plane, beside the ones in figure 6 ar mentioned parabolas as escape orbits for comets from a central sun, a line for the Einstein equation mc ${ }^{2}=$ hf, points for $\mathrm{x}^{2}+\mathrm{y}^{2}=0$ where Higgs can set a mass at a barycenter.

\section{Conclusion}

For teahing and technical modelling new tools and material is invented in order to demonstrate quanta facts which differ from the macroscopic experiences. They should be applied in the nanotehcnical range and demonstrated in courses for MINT/STEM education. For higher dimensional, projective gravitational fields can be consulted, for physical notations.

\section{References}

[1] Kalmbach G. Orthomodular Lattices. - London New York: Academic Press. 1983; 390.

[2] G Kalmbach HE. MINT-WIGRIS, MINT Verlag, Bad Woerishofen. 2017.

[3] G Kalmbach HE. (Chef-Hrsg), MINT (Mathematik, Informatik, Naturwissenschaften, Technik), MINT Verlag, Bad Woerishofen. 1997-2020; 1-65.

[4] Internet video under YouTube: Moebius Transformations Revealed. 2014.

[5] G Kalmbach U. Eberspaecher, MINT-Wigris Tool Bag, Bad Woerishofen. 2019.

[6] G Kalmbach, MINT-Wigris Postulates, in: researchgate.net under MINT-Wigris Project. 2020.

[7] E Schmutzer, Projektive einheitliche Feldtheorie, Harry Deutsch, Frankfurt. 2004.

[8] K Stierstadt, Physik der Materie, VCH, Weinheim. 1989.

[9] MINT-Wigris project (G Kalmbach HE) in the internet under: researchgate.net. 
[10] T Poston, I Stewart. Catastrophe theory and its applications, Pitman, London. 1978.

[11] G Kalmbach HE. A Heisenberg c-compass, J. of Applied Material Science \& Engineering Research. 2020; 4(2): 6367.

[12] G Kalmbach HE. Nucleons Strong Interaction SI-rotor, to appear.

[13] JMT Thompson, GW Hunt, A bifurcation theory for the instabilities of optimization and design, in: D. Berlinski (ed.), Mathematical Methods in the Social Sciences. 1979.

[14] JMT Thompson, ZA. Gaspar, A buckling model for the set of umbilic catastrophes (preprint, Eng. Dept. Univ. College, London). 1977.

[15] JMT Thompson, GW Hunt, General Theory of Elastic Stability, Wiley NewYork. 1973.

[16] GM Bell, DA Lavis, Thermodynamic phase changes and catastrophe theory, preprint. 1978.

[17] HE Stanley, Introductionto Phase transitions and critical phenomina, Oxford Univ. Press, London. 1971.

[18] A. Wald, On some systems of equations of mathematical economics, Econometrica. 1957; 19: 368-408.

[19] IN Howard, Bifurcations in reaction-diffusion problems, Adv. Math. 1975; 16: 246-258.

[20] DRJ. The buckling beam, in: A. Manning (ed.), Dynamical Systems, Springer, Berlin. 1975; 86-91.

[21] MV Berry, Waves and Thom's theorem, Adv. Physics. 1976; 25: 1-25. 